International Journal of Distributed and Parallel Systems (IJDPS) Vol.3, No.4, July 2012

\title{
Distributed Asynchronous Game Theoretic Solutions for Precoding Strategies in Multiuser MIMO Systems
}

\author{
Brima Fallah ${ }^{1}$, BenXiong Huang ${ }^{2}$ and Lai $\mathrm{Tu}^{3}$ \\ ${ }^{1,2,3}$ Department of Electronics and Information Engineering, \\ Huazhong University of Science and Technology, \\ Wuhan, Hubei, 430074 China. \\ ${ }^{1}$ brimaf2006@gmail.com, ${ }^{2}$ huangbx@hust.edu.cn, ${ }^{3}$ tulai.net@gmail.com
}

\begin{abstract}
In this paper, we proposed and illustrated a distributed low-complex algorithm for precoding strategy selection in a multi-user Multiple-input Multiple-output (MU-MIMO) wireless system using noncooperative games. We formulate the game as a non-cooperative game for an asynchronous distributed system in multi-user MIMO system known as Partially asynchronous distributed algorithm (PADA) and the convergence (Nash Equilibrium (NE) point) of the game. Mathematical framework providing sufficient conditions guaranteeing the uniqueness of the $N E$ and the convergence of the proposed algorithm is presented. (NE) which possess at least one pure strategy Nash equilibrium (NE) and the optimal strategy profile which maximizes the sum rate provides the solution for the existence and uniqueness of the game. The main focus is on non-cooperation of interference multi-user MIMO channel. Analysis of the proposed partially asynchronous distributed low-complex algorithm for precoding strategy selection in a multi-user MIMO wireless system using non-cooperative games is illustrated.
\end{abstract}

\section{KEYWORDS}

Precoding, Game theory, Multiuser, MIMO, Partially asynchronous

\section{INTRODUCTION}

Many recent works have investigated the study of interference channels using informationtheoretic principles [1][2][3]. However, game theory application to the study of interference channels has posed a significant research interest over the years.

Precoding strategy for multi-user (MU) multiple-input multiple-output (MIMO) systems is of significant importance and has received great research interest in recent years [4]. A centralized low-complexity algorithm for antenna selection not suitable for other limited feedback precoding techniques has been investigated in [5][6]. A MU-MIMO systems where heterogeneous users wish to dynamically share the restricted electromagnetic spectrum that rendered the use of radio resources as scarce with receiver having no CSI of the users pose a huge problem for the centralized low-complexity algorithms proposed in [5][6]. An efficient distributed precoding strategy selection algorithm with low-complexity is required to address the issues posed by the centralized approach in an heterogeneous users environment.

DOI : $10.5121 /$ ijdps.2012.3414 
International Journal of Distributed and Parallel Systems (IJDPS) Vol.3, No.4, July 2012

Distributed algorithms in MIMO system was research on three major schemes used to compute the Nash equilibria of a strategic non-cooperative game based on the updating characteristics of the users in the system. It is observed that these schemes are originally described in which each player optimizes iteratively its own payoff function following a prescribed updating schedule [11]. Jacobi Scheme [9][12][13] where all components are updated simultaneously, Gauss-Seidel scheme [7][8][10], all components are updated sequentially and totally asynchronous scheme [11][13][14], all components are updated in a totally asynchronous way, via a defined mapping based on water-filling based algorithms. [9] investigated the gradient-projection based algorithms as a distributed algorithm.

This paper only addresses the non-cooperation of interference multi-user MIMO channel. Analysis of the proposed partially asynchronous distributed low-complex algorithm for precoding strategy selection in a multi-user MIMO wireless system using non-cooperative games is illustrated. Formulation of the non-cooperative game model for a partially asynchronous distributed algorithm in multi-user MIMO system and analysis of the convergence (NE point) of the non-cooperative multi user MIMO system game were also illustrated.

\section{SYSTEM MODEL}

This section described the model used in this paper. An N-user MIMO equipped with $N_{r}$ antennas at the receiver and $N_{t_{i}}$ transmit antennas for user $i$, where $N=\{1, \ldots, n\}$ is considered. Let $H_{i j}$ denote the $M_{r} \times N_{t_{i}}$ channel matrix between transmitter $i$ and receiver $j$ with each entry modeled as independent identically distributed (i.i.d.) zero-mean unit-variance circularly symmetric complex Gaussian. We assume that the number of users and the channel matrices $\left\{H_{i j}\right\} \forall i, j \in P$ are fixed during each transmission block and change independently from one block to another obeying the ergodic random processes. The signal model of an $\mathrm{N}-$ user (MIMO) interference channel at receiver $i$ can be written as:

$$
y_{i}=H_{i i} G_{i} x_{i}+\sum_{i \neq j}^{n} H_{i j} G_{i} x_{i}+z \quad i=\{1,2 \ldots . N\}
$$

where $y_{i}$ is a $N_{r_{i}} \times 1$ channel output with a precoding strategy selection, $x_{i}$ is a $L_{i} \times 1$ information symbol block of user $i$. Note that $L_{i}$ is the number of transmission data substreams of user $i . G_{i}$ is the $M_{r} \times N_{t_{i}}$ precoding matrix of user $i$ and $z$ is a $N_{r} \times 1$ vector of additive white Gaussian noise (AWGN) with zero mean and covariance matrix equal to $I_{N_{r}}$ ie $E\left\{n_{i}, n_{i}^{H}\right\}=\sigma_{i}^{2} I_{N_{r}}$ with the assumption that information symbols are uncorrelated and with variance $\sigma_{i}^{2}$. For each user $i$, The code-book $\mathrm{M}_{i}=\left\{G_{i 1}^{*}, \ldots, G_{i k}^{*}, \ldots, G_{i K_{i}}^{*}\right\}, \forall i \in P$, is a finite set of precoding strategies (matrices) for all the supported modes, where $G_{i k}^{*}$ is the $k^{\text {th }}$ strategy of user $i$ and $K_{i}$ is the cardinality of $\mathrm{M}_{i}$. 
International Journal of Distributed and Parallel Systems (IJDPS) Vol.3, No.4, July 2012

\section{GAME-THEORETIC PRECODING STRATEGY}

\subsection{Problem Formulation And Game Model}

A system of low complexity receivers with no interference cancelation capabilities and the receivers treat the interference from other users as additive spatial noise is consider since we are interested in designing a decentralized algorithm. The above is applicable to the distributed algorithms that do not require neither a centralized control nor a coordination among the users.

Definition 1: Let $P=\left(P_{1}, P_{2}, \ldots, P_{N}\right)$ be set of players in the game and the codewords $G_{i}=\left\{G_{i 1}^{*}, \ldots, G_{i k}^{*}, \ldots, G_{i K_{i}}^{*}\right\}, \forall i \in P$ a discrete strategy space. That is, to each player $P_{i} \in P$, we may attribute a certain discrete set of strategies $G_{i}$. Note that $G_{i}$ is not always discrete in some game theory approach.

In our game model, the wireless users are considered as players. The users are assumed to be selfish and strictly rational agents, they will compete to maximize their individual mutual information with multiuser interference treated as noise [15]. The choices of precoding matrices are the users strategies and the corresponding transmission information rates of these players are considered as their payoffs denoted as;

$$
\bigcup_{i}=\log \operatorname{det}\left(\left|I+G_{i}^{H} H_{i i}^{*} R_{-i}^{-1} H_{i i}^{T} G_{i}\right|\right) \forall i \in\{1,2\}
$$

where $R_{-i}=\sigma_{i}^{2}+\sum_{j \in P, j \neq i} H_{j i}^{T} G_{j} G_{j}^{H} H_{j i}^{*}$ is the noise plus interference for user $i$.

Based on the above motivation, we can now define the payoff for user $i$ denoted as $\bigcup_{i}$ by the following;

$$
\bigcup_{i}\left(G_{i}, G_{-i}\right)
$$

Therefore considering the users precoding strategies $G_{i}$ as discrete and payoffs $\cup_{i}$, a discrete non-cooperative game in a normal form is denoted as

$$
\mathrm{G}_{0}=\left(P, G, \mathrm{U}_{i}\right)
$$

Remark 1: The above game illustrate the concept that the precoding strategies and payoffs make up a discrete non-cooperative game.

Let us now formally represent $G_{1}$ as a NE non-cooperative game. $G_{1}$ can be formally represented as:

$$
\left.\mathrm{G}_{1}\right): \max _{G_{i} \in \mathrm{M}_{i}}=\mathrm{U}_{i}\left(G_{i}, G_{-i}\right) \quad \forall i \in P
$$

where $U_{-i}$ stands for the combination of precoding vectors of all users except user $i$.

\subsection{Non-Cooperative Game And Nash Equilibrium Solution}

In this subsection, we analyze the $\mathrm{NE}$ of the above proposed game. In the non-cooperative game, where users are selfish and competing with each other for the limited available resources, 
International Journal of Distributed and Parallel Systems (IJDPS) Vol.3, No.4, July 2012

the result of the game will be a Nash equilibrium that satisfies the following theorem.

Definition 2: Let $\mathrm{G}=\left(P, G, \bigcup_{i}\right) \forall i \in P$ be a $P$ player game in normal form. Then $\mathrm{G}$ has at least one Nash equilibrium.

Remark 2: $\mathrm{G}_{1}$ is a potential game which has at least one pure strategy NE and the optimal solution of the sum rate maximization problem as:

$$
\left(\mathrm{P}_{1}\right): \max _{G_{i} \in \mathrm{M}_{i}} \mathrm{U}_{\Omega}=\log \operatorname{det}\left(\left|I+G_{i}^{H} H_{i i}^{*} R_{-i}^{-1} h_{i i}^{T} G_{i}\right|\right) \forall i \in\{1,2\}
$$

constitutes a pure strategy $\mathrm{NE}$ of $\mathrm{G}_{1}$.

Let $\mathrm{G}^{C}=\left(P, G, \mathrm{U}_{i}^{C}\right) \forall i \in N$ and the payoff function of each user $i$ be $\mathrm{U}_{i}^{C}=\mathrm{U}_{\Omega}$. Also, let $\left(G_{i}^{o}, \ldots, G_{n}^{o}\right)$ denote the optimal solution of $P_{1}$ that maximized $\bigcup_{\Omega}$ Therefore, $G_{i}^{\prime} \in G_{i}$, $G_{i}^{\prime} \neq G_{i}^{o} \forall i \in N$ is an alternate strategy of user $i$ that boils down to the following:

$$
\mathrm{U}_{i}^{C}\left(G_{i}^{\prime}, G_{-i}^{0}\right) \leq \mathrm{U}_{i}^{C}\left(G_{i}^{o}, G_{-i}^{0}\right)
$$

Equation (7) illustrate the simple fact that if a user changes its own strategy independently, its payoff can never be higher than the payoff obtained by $\left(G_{i}^{o}, \ldots, G_{n}^{o}\right)$. Therefore, the strategy profile $\left(G_{i}^{o}, \ldots, G_{n}^{o}\right)$ is a pure strategy NE of $\mathrm{G}^{C}[16]$.

Let $G_{i}^{\prime} \in G_{i}$ be an alternative strategy of user $i$. Therefore,

$$
\begin{aligned}
& \bigcup_{i}\left(G_{i}, G_{-i}\right)-\bigcup_{i}\left(G_{i}^{\prime}, G_{-i}\right)=\log \operatorname{det}\left(\left|I+G_{i}^{H} H_{i i}^{*} R_{-i}^{-1} H_{i i}^{T} G_{i}\right|\right) \\
& -\log \operatorname{det}\left(\left|I+\left(G_{i}^{H} H_{i i}^{*} R_{-i}^{-1} H_{i i}^{T} G_{i}\right)^{\prime}\right|\right) \\
& =\bigcup_{\Omega}\left(G_{i}, G_{-i}\right)-\bigcup_{\Omega}\left(G_{i}^{\prime}, G_{-i}\right)
\end{aligned}
$$

Using simple substitution of the rate sum $R_{\Omega}$ by a potential function $\Phi$, then (8) can be formulated as

$$
\forall G_{i} G_{i}^{\prime}, \bigcup_{i}\left(G_{i}, G_{-i}\right)-\bigcup_{i}\left(G_{i}^{\prime}, G_{-i}\right)=\Phi\left(G_{i}, G_{-i}\right)-\Phi\left(G_{i}^{\prime}, G_{-i}\right)
$$

Game $G_{1}$ satisfies the properties of a potential game defined in [17] with the sum rate as the potential function. NEs of $G_{1}$ are similar to the set of NEs of $G^{C}$ which illustrates without generality that $\left(G_{i}^{o}, \ldots, G_{n}^{o}\right)$ is also the pure strategy NE of $\mathrm{G}_{1}$.

\section{DISTRIBUTED PRECODING STRATEGY SELECTION ALGORITHM}

\subsection{Partially Asynchronous Algorithm Model [11]}

Let $\Omega_{1}, \Omega_{2}, \ldots \Omega_{N}$ be the given subsets of Euclidean spaces and let $\Omega=\Omega_{1} \times \Omega_{2} \times, \ldots \times \Omega_{N}$ be their Cartesian product. Consequently, the mappings $F_{i}: \Omega \mapsto \Omega_{i}, i=1, \ldots, N$ are given and elements of $\Omega$ are decomposed into block-components, that $G=\left(G_{1}, G_{2}, \ldots G_{N}\right)$ with $G_{i} \in \Omega_{i}$ . We consider the following partially iterative algorithm shown in Table 1: 
International Journal of Distributed and Parallel Systems (IJDPS) Vol.3, No.4, July 2012

Table 1: Distributed Partially Asynchronous Algorithm

\begin{tabular}{c}
\hline Distributed Precoding Strategy Selection Algorithm \\
when $n \leq 0$ \\
$G_{i}=F_{i}\left(G_{1}^{\tau_{1}^{i}(n)}\right) \quad i=1,2, \ldots N$ \\
when $n \geq 0:$ \\
$G_{i}^{n+1}=G_{i}^{(n)} \quad \begin{array}{c}\text { if } n \notin \mathrm{T}_{i} \\
G_{i}^{n+1}=\end{array} F_{i}\left(\left(G_{1}^{\tau_{1}^{i}(n)}, G_{2}^{\tau_{2}^{i}(n)}, \ldots, G_{N}^{\tau_{N}^{i}(n)}\right) \quad\right.$ if $n \in \mathrm{T}_{i}$ \\
\hline
\end{tabular}

$\tau_{r}^{i}(n)$ is the newly and immediate updated time at which the interference from user $r$ is decerned by user $i$ at the $n^{\text {th }}$ iteration and $\mathrm{T}_{i}$ is the set of times $n$ at which $\tau_{r}^{i}(n)$ is updated. For the above algorithm to hold,$\tau_{r}^{i}(n)$ and $\mathrm{T}_{i}$ must satisfy the following assumptions.

Assumption 1: (Partial Asynchronism) There exists a positive integer $B$ such that:

(a) For every $i$ and for every $n \geq 0$, at least one of the elements of the set $\{n, n+1, \ldots, n+B-1\}$ belongs to $\mathrm{T}_{i}$.

(b) There holds $\left(n-B<\tau_{r}^{i}(n) \leq n\right.$, ) for all $r$ and $i$, and all $n \geq 0$ belonging to $\mathrm{T}_{i}$.

(c) There holds $\tau_{i}^{i}(n)=n$ for all $i$ and $n \in \mathrm{T}_{i}$.

\subsection{The Convergence Theory}

We consider a partially asynchronous iteration of the form $G:=F(G)$ where $F(G)=\left(F_{1}(G), \ldots, F_{N}(G)\right)$ and $F:=\mathrm{R}^{n} \mapsto \mathrm{R}^{n}$. We let $\Omega^{*}:=\left\{G \in \mathrm{R}^{n} \mid F(G)=G\right\}$ be the set of fixed points of $F$, with the following properties:

Assumption 2: Assumed that:

(a) The set $\Omega^{*}$ is nonempty and convex.

(b) The function $F$ is continuous.

(c) The function $F$ is non-expansive, that is, it satisfies

$\left\|F(G)-G^{*}\right\|_{\infty} \leq\left\|G-G^{*}\right\|_{\infty}, \forall G \in \mathrm{R}, \forall G^{*} \in \Omega^{*}$

For any $G \in \mathrm{R}^{n}$, we denote by $G\{G)$ the distance of $G$ from $\Omega^{*}$, defined by

$$
G(G)=\inf _{G^{*} \in \Omega^{*}}\left\|G-G^{*}\right\|_{\infty}
$$

The following preliminary result will be needed in the sequel. 
Proposition 1: Suppose that $F:=\mathrm{R}^{n} \mapsto \mathrm{R}^{n}$ satisfies Assumption 1. Then:

(a) The set $\Omega^{*}$ is closed.

(b) For every $G \in \mathrm{R}^{n}$, there exists some $G^{*} \in \Omega^{*}$ such that $G(G)=\left\|G-G^{*}\right\|_{\infty}$.

(c) The function $g: \Omega \mapsto \mathrm{R}^{n}$ is continuous.

(d) For every $G \in \mathrm{R}^{n}$ we have $G(F(G)) \leq G(G)$.

\section{Assumption 3:}

(a) The set $\Omega^{*}$ is convex.

(b) For every $G \in \mathrm{R}^{n}$ and $G^{*} \in \Omega^{*}$ such that $G(\mathrm{X})=\left\|G-G^{*}\right\|_{\infty}>0$, there exists some $i$ such that $\left\|G_{i}-G_{i}^{*}\right\| \leq\left\|G-G^{*}\right\|_{\infty}$ and $Z_{i}(G) \neq G_{i}$.

(c) If $G \in \mathrm{R}, F_{i}(G) \neq G_{i}$, and $G^{*} \in \Omega^{*}$, then $\left\|F(G)-G_{i}\right\|<\left\|G-G^{*}\right\|_{\infty}$

We can see that Assumption 3(b) and 3(c) are closely related to the contraction property of the function $F$. Assumption 3(c) illustrate the fact for certain algorithms involving a relaxation parameter.

Proposition 2: Suppose that a function $\mathrm{H}: \mathrm{R} \mapsto \mathrm{R}$ satisfies Assumption 2, 3(a) and 3(b). Let $\alpha \in(0,1)$, then the mapping $\mathrm{Z}: \mathrm{R} \mapsto \mathrm{R}$ defined by

$$
F(G)=\alpha G+(1-\alpha) \mathrm{H}(G)
$$

satisfies Assumption 2 and 3. The following result is achieved:

Theorem 2: Suppose that $F:=\mathrm{R}^{n} \mapsto \mathrm{R}^{n}$ satisfies Assumption 2 and 3, and suppose that Assumption 1 (Partial Asynchronism) holds. Then the sequence $G(n)$ generated by the asynchronous iteration $G:=F(G)$ converges to some element of $\Omega^{*}$.

\subsection{Main Algorithm}

It is now time to formulate the algorithm used in this paper. The following definitions are used to illustrate the algorithm:

(a) $G_{-i}^{\left(\tau^{i}(n)\right)}=\left(G_{1}^{\left(\tau_{1}^{i}(n)\right)}, \ldots, G_{i-1}^{\left(\tau_{i-1}^{i}(n)\right)}, G_{i+1}^{\left(\tau_{i+1}^{i}(n)\right)}, \ldots, G_{G}^{\left(\tau_{G}^{i}(n)\right)}\right)$

(b) $G_{i}^{(n)}$ is the covariance matrix of the vector signal transmitted by user $i$ at the $n^{\text {th }}$ iteration.

(c) $\mathrm{T}_{i}=\mathrm{T}$ is the set of times $n$ when $G_{i}^{(n)}$ is updated.

(d) $\tau_{r}^{i}(n)$ is the most recent time when the interference from user $r$ is perceived by user $i$ at the $n^{\text {th }}$ iteration. 
Table 2: Distributed Precoding Strategy Selection Algorithm

\begin{tabular}{|c|}
\hline Distributed Precoding Strategy Selection Algorithm \\
\hline$G_{i}^{0}=$ any feasible covariance matrix: \\
for $n=0: N_{i t}$ \\
$G_{i}=F_{i}\left(G_{i}^{\tau_{1}^{i_{1}(n)}}\right) \quad i=1,2, \ldots N$ \\
when $n \geq 0:$ \\
$G_{i}^{n+1}=G_{i}^{(n)} \quad \begin{array}{l}\text { if } n \notin \mathrm{T}_{i} \\
G_{i}^{n+1}=F_{i}\left(\left(G_{1}^{\tau_{1}^{i}(n)}, G_{2}^{\tau_{2}^{i}(n)}, \ldots, G_{N}^{\tau_{N}^{i}(n)}\right) \quad \text { if } n \in \mathrm{T}_{i}\right.\end{array}$ \\
\hline
\end{tabular}

\section{SIMULATION RESULTS}

In this section, we use a computer simulations to evaluated the performance of the proposed algorithm. Each link is modeled as a 4-transmitter and 4-receiver structure based on the following assumptions: A Gaussian interference channel with $N=4$ links communicating through a $N_{t}$ and $N_{r}$ MIMO channel, a spatially i.i.d flat fading channel is assumed whose channel coefficient matrix for a user $i, H_{i}$ is given by $N_{t_{i}} \times N_{r}$, partial channel state information (CSI) be available at the transmitter and receivers of each link, a system with low complexity receivers with no interference cancelation capabilities, the receivers treat the interference from other users as additive spatial noise, the channel matrices $H_{i i}$ are square nonsingular.

We employed the PADA procedure to perform the entire simulation.asynchronous parameter $B$ is a discrete value; for example 1,15 . 
International Journal of Distributed and Parallel Systems (IJDPS) Vol.3, No.4, July 2012

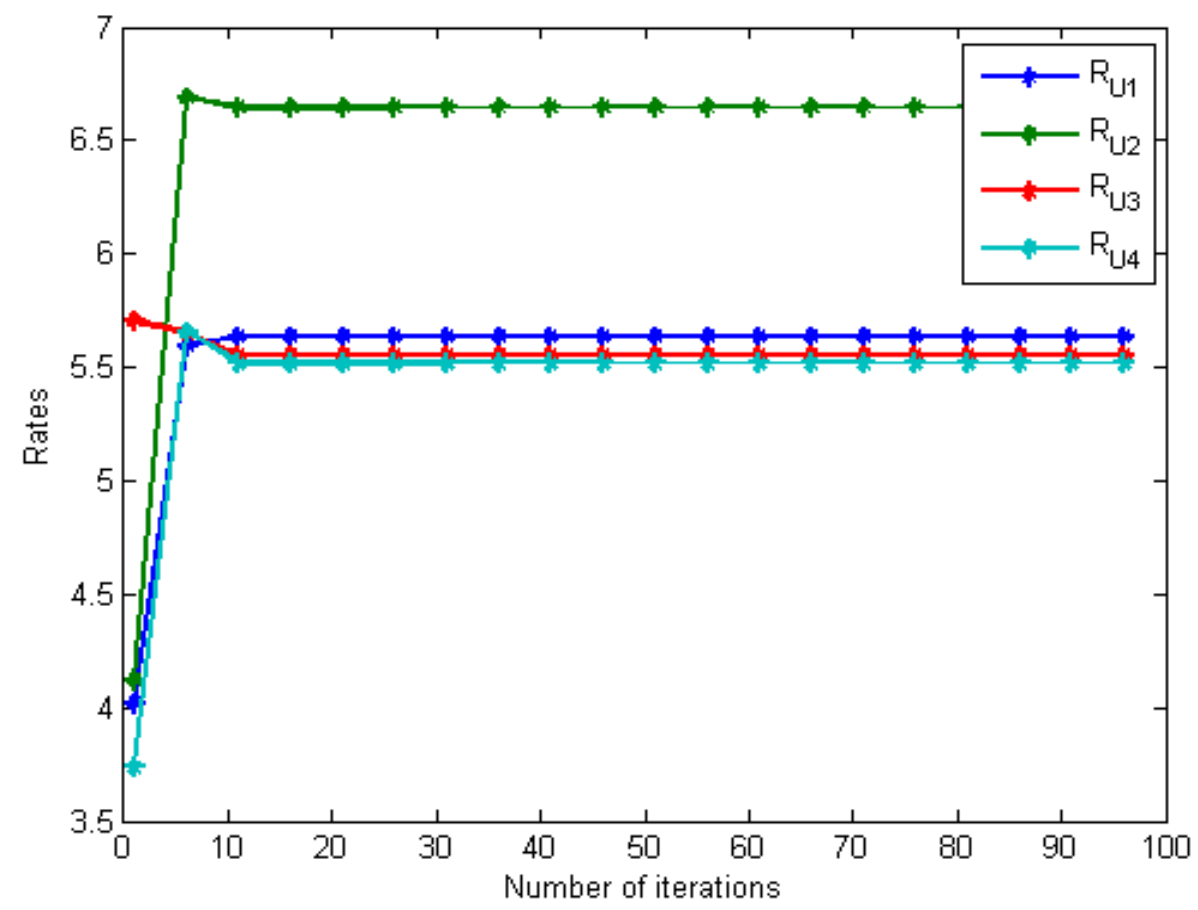

Figure 1: Comparison of rate performance for $\mathrm{B}=1$.

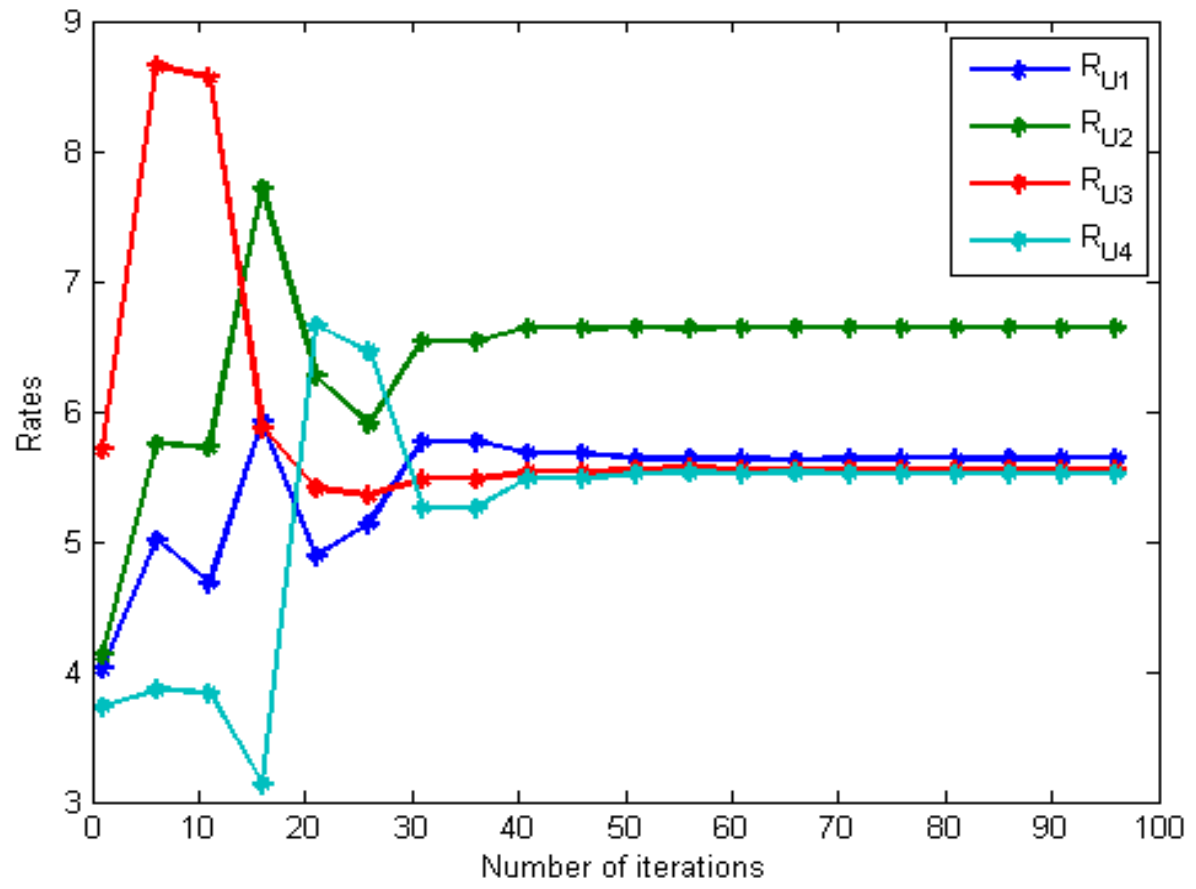

Figure 2: Comparison of rate performance for $B=15$. 
International Journal of Distributed and Parallel Systems (IJDPS) Vol.3, No.4, July 2012

From Fig. 1, 2, it is shown that the performance of asynchronous parameter $(B=1)$ is better than the performance of asynchronous parameter $(B=15)$. This is because multiple NEs exist in most situations and it is very likely for PADA to miss the optimal (or near-optimal) NE when becomes large. For PADA, it should be expected that the larger is the value of asynchronous parameter, the worse the performance becomes.

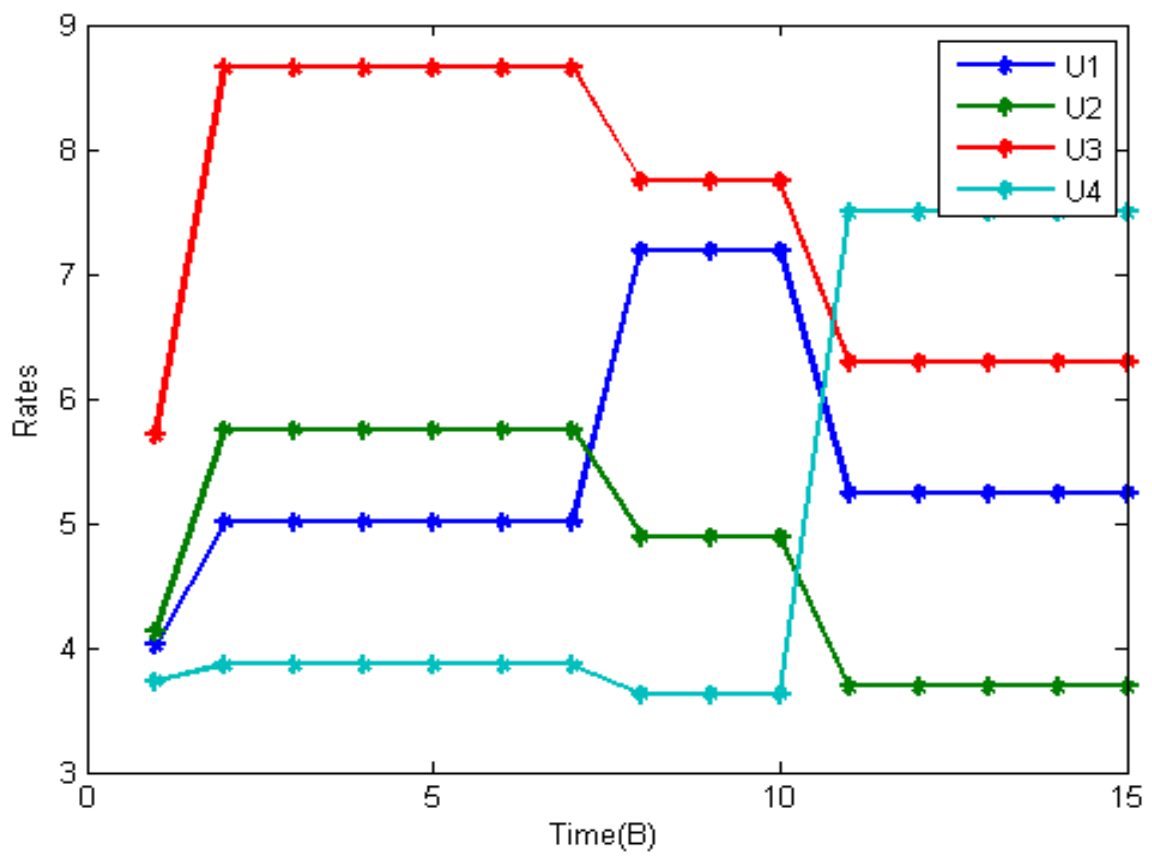

Figure 3: Comparison of rate performance for $\mathrm{B}=1-15$

From Figs. 3, we find that the proposed adaptive asynchronous parameter design mechanism can show a good balance between rate performance and convergence speed for the average rate for each user from 1 to 15 asynchronous parameters. These results shows that the adaptive asynchronous mechanism is faster and efficient.

Practically, we can regulate the value of the asynchronous parameter or use adaptive asynchronous mechanism to trade off the performance and the complexity according to the channel conditions and the requirement of the system designer.

As the value of $B$ increases, the time for convergency also increases. As long as the system stays long to converge, users will make use of the outdated information. The overall systems convergence speed cripples due to the characteristics information update of the strategy of some other users. Based on the above illustration, PADA is flexible and efficient for any precoding strategy in MU-MIMO system.

\section{CONCLUSION}

In this paper, we studied the distributed precoding strategy selection for MU-MIMO through a game theoretic approach. A decentralized learning algorithm, partially asynchronous algorithm is proposed to obtain the NE. We have illustrated the concept behind the partially asynchronous iterative distributed algorithm(PADA). We formulated sufficient conditions guaranteeing the 
International Journal of Distributed and Parallel Systems (IJDPS) Vol.3, No.4, July 2012

convergence and uniqueness of the algorithm that represent a set of conditions for the convergence of MU-MIMO non-cooperative game theoretic precoding strategy. A pure strategy $\mathrm{NE}$ in our proposed game and the social optimal strategy profile constitutes for an NE.

We also show by simulation that PADA converge to a Jacobi Scheme also know as simultaneous asynchronous iterative algorithm when the asynchronous criteria is very small eg. $B=1$. When the time convergence criterion $B$ is set to a higher value eg. $B=15$, it renders the system to a low speed convergence of the use of outdated information. Thus, $B$ must be a significantly small value for faster convergence of the proposed algorithm. A significant improvement can be achieved in the sum rate performance by repeating the proposed algorithm. To augment the tradeoff between the system performance and the convergence speed adaptive step size mechanism is considered in this findings.

Compared to the exhaustive search algorithms such as Jacobi and Gauss-Seidel, the proposed algorithm can achieve a comparable sum rate performance with low complexity.

\section{REFERENCES}

[1] M. H.M. Costa, “On the Gaussian interference channel”, IEEE Trans. Inf. Theory, vol. 31, pp. 607--615, Sep. 1985.

[2] G. A. El and T. M. Cover, "Multiple user information theory", Proc.IEEE, vol. 68, pp. 14661483, 1980.

[3] S. Vishwanath and S. A. Jafar, "On the capacity of vector Gaussian interference channels", in Proc. IEEE Workshop Information Theory 2004, pp. 365-369.

[4] D. J. Love et al., "An overview of limited feedback in wireless communication systems", IEEE J. Sel. Areas Commun., vol. 26, no. 8, pp.1341-1365, Oct. 2008.

[5] Y. Kim, S. Cho, and D. K. Kim, "Low complexity antenna selection based MIMO scheduling algorithms for uplink multiuser MIMO/FDD system", in Proc. IEEE VTC, 2007, pp. 1663-1667

[6] R. Chen, Z. Shen, J. G. Andrews, and R. W. Heath, Jr., "Multimode transmission for multiuser MIMO systems with block diagonalization”, IEEE Trans. Signal Process., vol. 56, no. 7, pp. 3294-3320, Jul. 2008.

[7] S. T. Chung, S. J. Kim, J. Lee, and J. Cioffi, "A game-theoretic approach to power allocation in frequency-selective gaussian interference channels", IEEE International Symposium on Information Theory, 2003. Proceedings., pp. 316-316, June-4 July 2003.

[8] Z.-Q. Luo and J.-S. Pang, "Analysis of iterative waterfilling algorithm for multiuser power control in digital subscriber lines”, EURASIP J. Appl. Signal Process., vol.2006, pp. 1-10, 2006.

[9] G. Scutari, D. Palomar, and S. Barbarossa, "Optimal Linear Precoding Strategies for Wideband Non-Cooperative Systems Based on Game Theory-Part II: Algorithms”, IEEE Transactions on Signal Processing, vol. 56, no. 3, p. 1250, 2008.

[10] G. Scutari, D.P. Palomar, and S. Barbarossa, "The MIMO Iterative Waterfilling Algorithm", Signal Processing, IEEE Transaction, accepted for publication.

[11] D. Bertsekas and J. Tsitsiklis, "Parallel and Distributed Computation”, Old Tappan, NJ (USA); Prentice Hall Inc., 1989.

[12] K. Shum, K. Leung, and C. Sung, "Convergence of iterative waterfilling algorithm for Gaussian interference channels”, IEEE Journal on Selected Areas in Communications, vol. 25, no. 6, pp. 1091-1100, 2007. 
International Journal of Distributed and Parallel Systems (IJDPS) Vol.3, No.4, July 2012

[13] G. Scutari, D. Palomar, and S. Barbarossa, "Simultaneous iterative water-filling for Gaussian frequency-selective interference channels", in Proc. of the 2006 IEEE International Symposium on Information Theory (ISIT 2006), 2006.

[14] G. Scutari, D. Palomar, and S. Barbarossa, "Distributed Totally Asynchronous Iterative Waterfilling for Wideband Interference Channel with Time/Frequency Offset", in Proc. of the IEEE Int. Conf. on Acoustics, Speech, and Signal Processing (ICASSP), Honolulu, Hawaii, USA, 2007.

[15] Wei Zhong, Youyun Xu, Meixia Tao, Yueming Cai, "Game Theoretic Multimode Precoding Strategy Selection for MIMO Multiple Access Channels", IEEE Signal Processing Letters, Vol. 17, No. 6, June 2010.

[16] R. Myerson, "Game Theory: Analysis of Conflict", Cambridge, MA:Harvard Univ. Press, 1991.

[17] D.Monderer and L. S. Shapley, "Potential games", Games and Economic Behavior, vol. 14, pp. 124-143, 1996.

\section{Authors:}

Brima Fallah received his B. Eng. and M. Eng. degrees from Huazhong University of Science and Technology (HUST), Wuhan, Hubei, P. R. China in 2007 and 2009 respectively. From 2009 to 2012, he is a Doctoral student at HUST majoring in the field of Communication and Information Engineer. His research interests include Resource management in Wireless and Mobile Networks, Multiple Antennas Technologies (MIMO), Game Theory, Wireless Network, Cooperative Relay Communications and Cognitive Radio.

Email address: brimaf2006@gmail.com

Lai Tu received his $\mathrm{PhD}$ degree from Huazhong University of Science and Technology, Wuhan, Hubei, P. R. China. He is currently an associate professor at Department of Electronic and Information Engineering, Huazhong University of Science and Technology, P. R. China. His research interests include vehicular adhoc networks, Sensor networks, next generation communication system and communication signal processing.

Email address: tulai.net@gmail.com

Benxiong Huang received his BS.C in 1987 and $\mathrm{PhD}$ in 2003 from Huazhong University of Science and Technology (HUST), Wuhan, Hubei, P. R. China. He is currently a professor in the Department of Electronic and Information Engineering at HUST. His research interests include next generation communication system and communication signal processing.

Email address: huangbx@ @ust.edu.cn

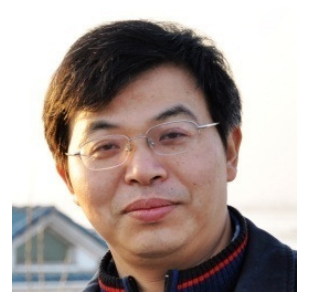

